\title{
Metastatic breast carcinoma with appearance resembling micropapillary ductal carcinoma in situ
}

\author{
A H S Lee, T P Telfer, R R Millis
}

ICRF Clinical

Oncology Unit, Second Floor,

New Guy's House,

Guy's Hospital,

London SE1 9RT

A H S Lee

R R Millis

BUPA Alexandra

Hospital,

Walderslade, Kent

T P Telfer

Correspondence to: Dr A H S Lee.

Accepted for publication 26 September 1994

\begin{abstract}
Aim-To investigate tumour in an axillary lymph node resembling micropapillary ductal carcinoma in situ.

Methods-Sections of tumour in the breast and axillary lymph node were stained with haematoxylin and eosin, and immunohistochemically with antibodies to basement membrane and myoepithelial cells.

Results-Tumour in both the breast and axillary lymph node contained areas resembling micropapillary ductal carcinoma in situ. Surrounding these islands, there was a band of eosinophilic material resembling basement membrane and spindle cells that in places appeared to lie outside the basement membrane. Micropapillary tumour at both sites showed weak and discontinuous staining for collagen IV and laminin. The spindle cells stained for $\alpha$-smooth muscle actin, but not for S100. By contrast, immunohistochemistry showed complete rings of basement membrane and myoepithelial cells around definite ductal carcinoma in situ and normal breast lobules and ducts.
\end{abstract}

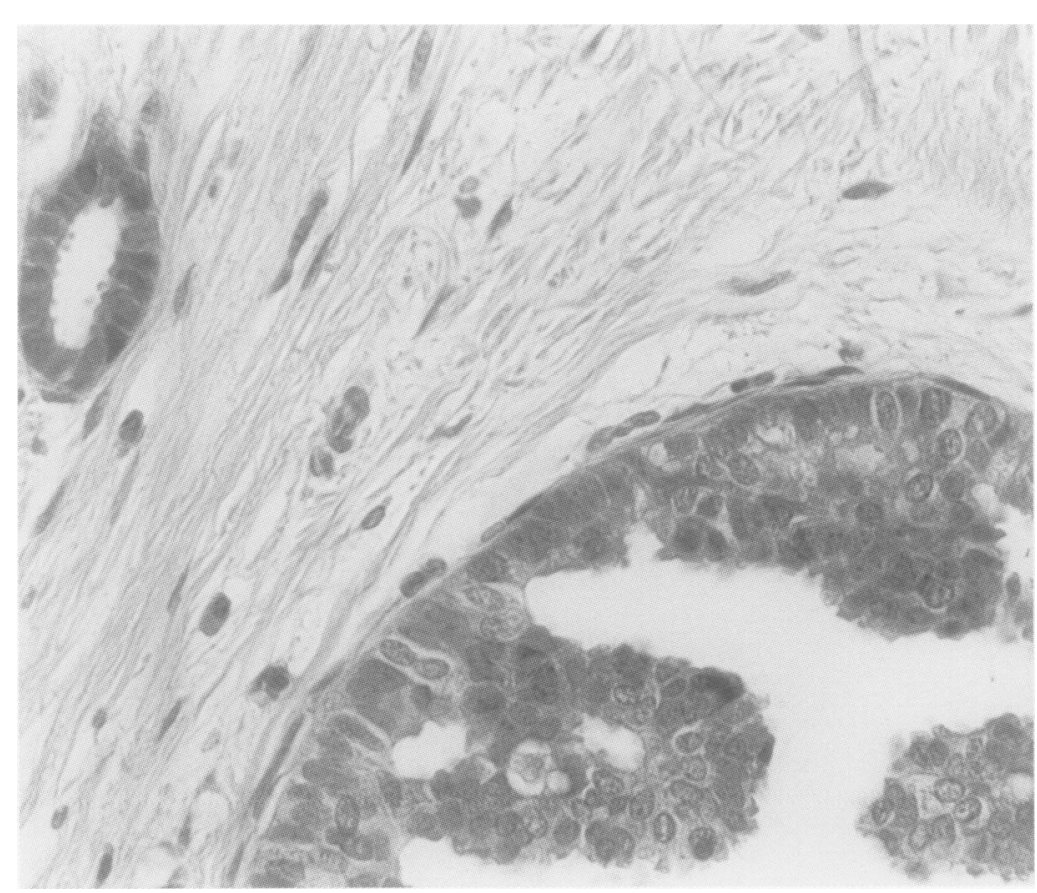

Figure 1 Invasive carcinoma in the breast showing tubular carcinoma and papillary carcinoma, the latter mimicking micropapillary ductal carcinoma in situ with spindle cells resembling myoepithelial cells around part of its circumference. Haematoxylin and eosin.
Conclusions-Invasive primary and metastatic carcinoma of the breast can have a growth pattern resembling micropapillary ductal carcinoma in situ.

(f Clin Pathol 1995;48:380-382)

Keywords: Invasive, metastasis, breast carcinoma, micropapillary.

It is well known that invasive primary and metastatic carcinomas of the breast can have growth patterns resembling comedo ${ }^{1}$ or cribriform ductal carcinoma in situ. ${ }^{2}$ However, invasive carcinoma of the breast resembling micropapillary ductal carcinoma in situ is not so well recognised, ${ }^{3}$ and we have not seen this pattern before in a metastasis.

\section{Case report}

A 53 year old woman with synchronous bilateral carcinoma of the breast was treated by bilateral modified radical mastectomy and adjuvant chemotherapy.

Macroscopic examination showed each breast to contain three separate ill-defined firm masses 1.5 to $2.5 \mathrm{~cm}$ in diameter. Histology of the right breast showed multiple foci of tubular carcinoma, one of which was contiguous with a fibroadenoma; and one small focus of grade III invasive ductal carcinoma (NOS). Focally, one of the areas of tubular carcinoma showed a micropapillary pattern resembling ductal carcinoma in situ (fig 1). There was associated definite in situ carcinoma mainly of ductal type with cribriform, solid and comedo patterns, composed of cells with small monomorphic

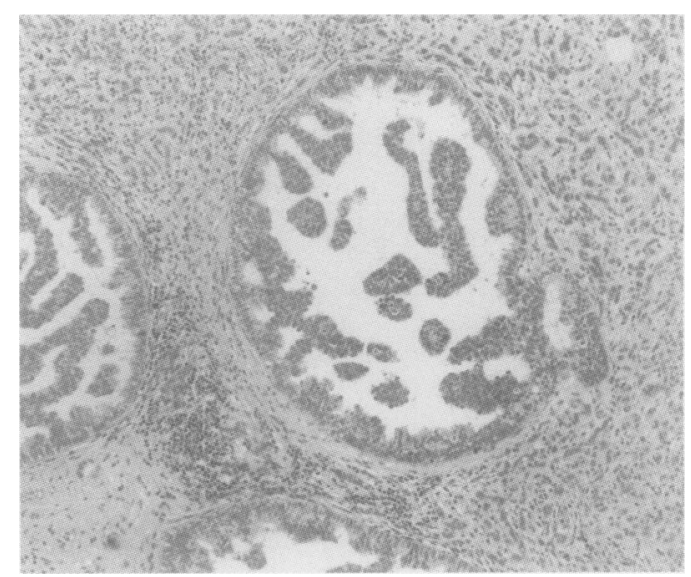

Figure 2 Metastatic carcinoma in axillary lymph node showing diffuse infiltation and micropapillary areas. Haematoxylin and eosin. 


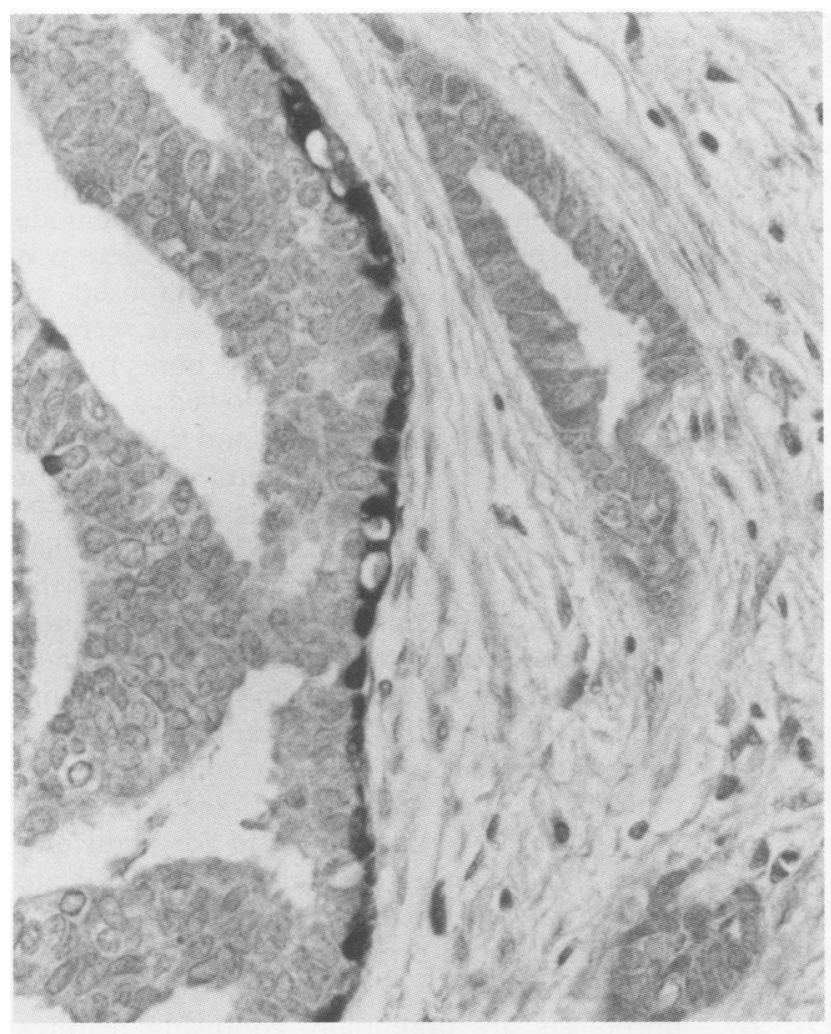

(A)

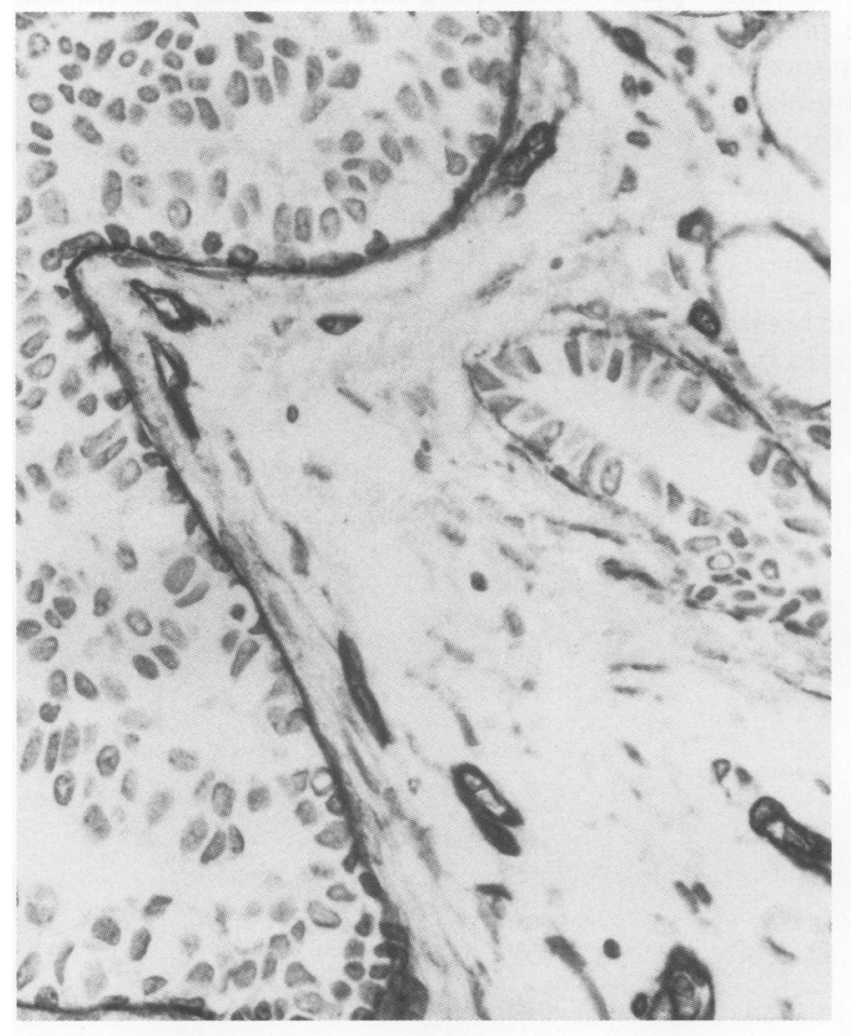

(C)

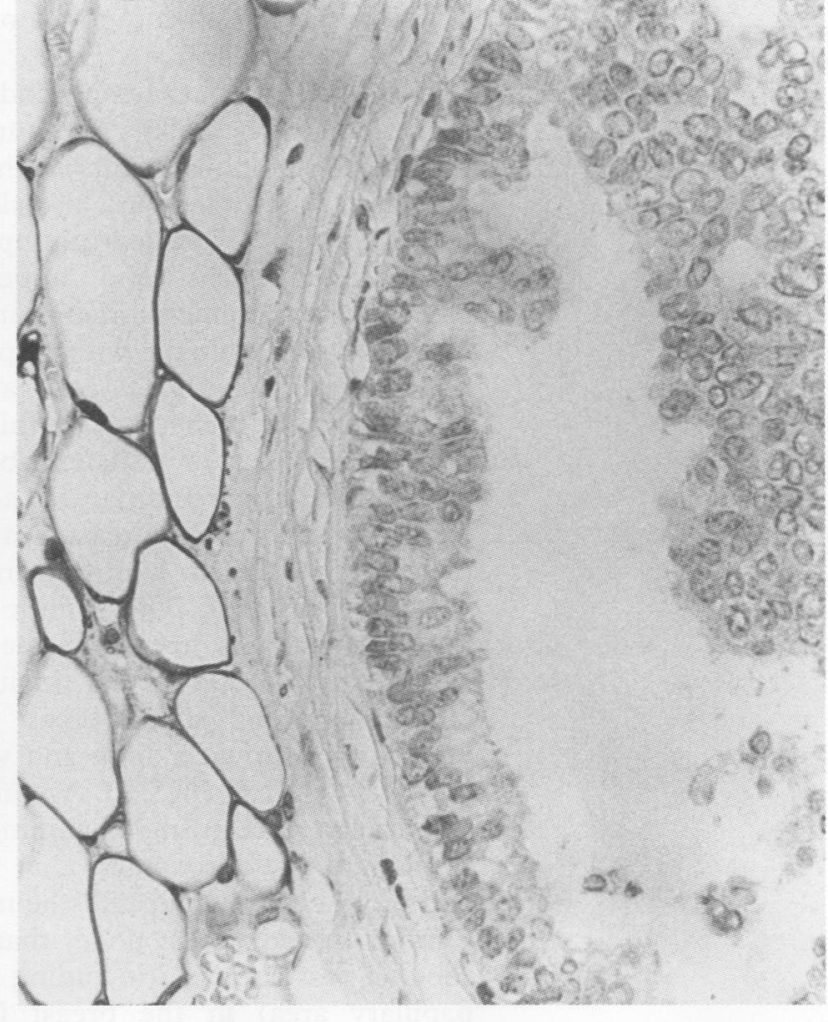

(B)

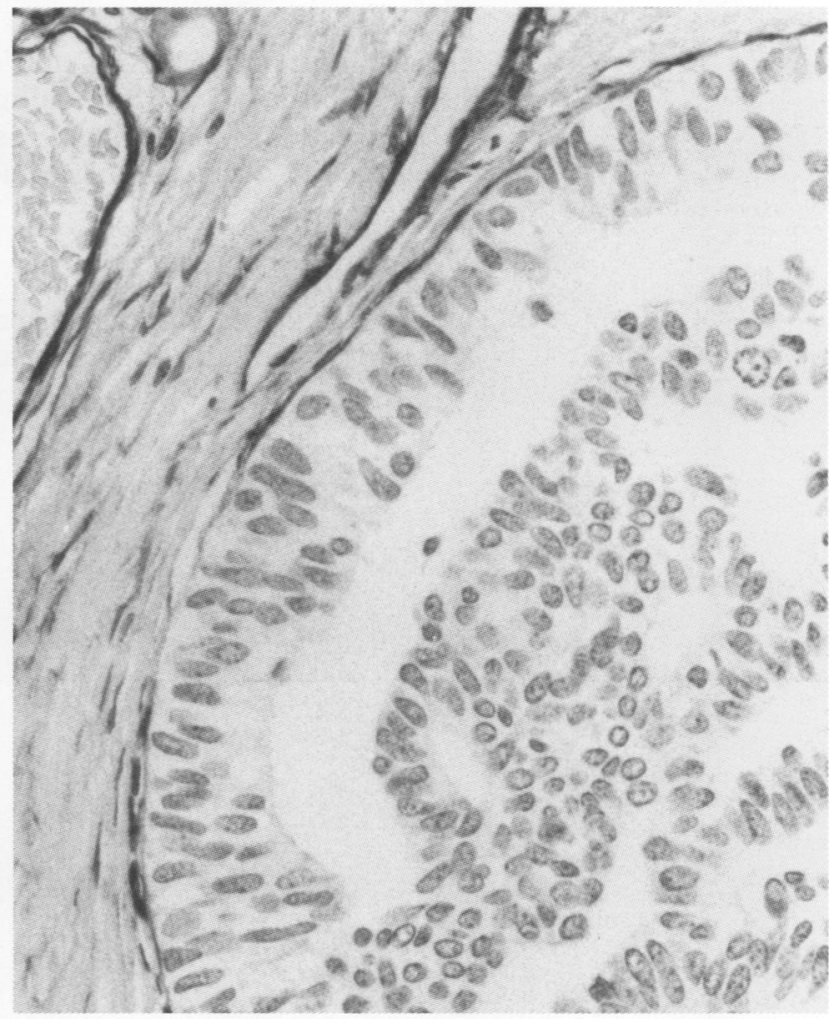

(D)

Figure 3 Myoepithelial cells staining for $S 100$ are present $(A)$ around the ductal carcinoma in situ, but not the tubular carcinoma in the breast, and (B) not around the micropapillary tumour in the lymph node. (C) There is a complete ring of staining for laminin around the ductal carcinoma in situ. By contrast, there is only patchy, weak staining around the tubular carcinoma in the breast $(C)$ and $(D)$ around the micropapillary tumour in the lymph node.

nuclei; and focal lobular carcinoma in situ. Two of 13 ipsilateral axillary lymph nodes contained metastatic deposits. Both were composed of diffuse sheets of tumour cells, but in part of one the appearance was strikingly similar to micropapillary ductal carcinoma in situ (fig 2).
In the left breast the foci of invasive carcinoma had features predominantly of lobular carcinoma, but with focal areas of ductal carcinoma (NOS) grade II. The in situ carcinoma on this side was also predominantly lobular. One of the 11 left axillary lymph nodes con- 
tained a metastasis composed of solid sheets of lobular carcinoma.

In the right axillary lymph node, around the islands of micropapillary tumour, there was a band of eosinophilic material that resembled basement membrane, and also slender spindle cells, but these were less plump than typical myoepithelial cells and appeared to lie outside the basement membrane. Immunohistochemical staining with a panel of antibodies to myoepithelial cells and basement membrane was carried out on this lymph node and on a section of ipsilateral breast that included tubular carcinoma with adjacent fibroadenoma and normal breast. An avidin-biotin technique was used, with trypsin predigestion where appropriate. The spindle cells around the micropapillary tumour in the lymph node, and the tubular carcinoma (including the micropapillary area) in the breast were positive for $\alpha$-smooth muscle actin and vimentin, and negative for S100 (fig 3B). Around both, there was an incomplete ring of staining for collagen IV, laminin and reticulin, that was stronger and more complete around the micropapillary tumour in the lymph node, than around the tubular carcinoma (including the micropapillary area) in the breast (fig 3D). By contrast, complete rings of basement membrane and myoepithelial cells ( $\alpha$-smooth muscle actin and S100 positive) were present around the normal lobules and carcinoma in situ (figs $3 \mathrm{~A}$ and $3 \mathrm{C}$ ). Stromal myofibroblasts in both sections were strongly positive for both vimentin and $\alpha$-smooth muscle actin.

\section{Discussion}

One area of the tubular carcinoma in the breast and part of one axillary node metastasis in this woman were strikingly similar to micropapillary ductal carcinoma in situ. A possible case of papillary carcinoma arising in benign epithelial inclusions in an axillary lymph node has been described. ${ }^{4}$ We felt, however, that primary ductal carcinoma in situ in the axillary lymph node was unlikely in this woman. Firstly, there was adjacent metastatic tumour composed of diffuse sheets of cells; secondly, there were no benign epithelial inclusions in the node; thirdly, the tumour in the breast showed a similar micropapillary pattern focally; and fourthly, the immunohistochemical staining pattern for basement membrane and myoepithelial cells was similar to that seen in the invasive tubular carcinoma with a micropapillary area in the breast, and different from that seen around carcinoma in situ in the breast. Although their exact position was difficult to define, in some places the spindle cells around the micropapillary foci in the node appeared to lie outside the basement membrane. This appearance, the positive staining for actin and vimentin, and negative staining for $S 100$ are consistent with these cells being stromal myofibroblasts. The presence of focal basement membrane and surrounding stromal myofibroblasts are potentially confusing features of this breast carcinoma. Awareness of this pattern of invasive carcinoma may prevent the erroneous diagnosis of carcinoma in situ.

1 Cowen PN. Recognition of intraduct mammary carcinoma [letter]. $\mathcal{F}$ Clin Pathol 1980;33:797.

2 Page DL, Dixon JM, Anderson TJ, Lee D, Stewart HJ Invasive cribriform carcinoma of the breast. Histopathology 1983;7:525-36.

3 Ahmed A. Diagnostic pathology of the breast: a text and colour atlas. Edinburgh: Churchill Livingstone, 1992:102-3.

4 Walker AN, Fechner RE. Papillary carcinoma arising from ectopic breast tissue in an axillary lymph node. Diagn Gynecol Obstet 1982;4:141-5. 